\title{
Isotopic cross sections of fragmentation residues produced by light projectiles on carbon near $400 \mathrm{~A} \mathrm{MeV}$
}

J. M. Boillos, ${ }^{1}$ D. Cortina-Gil $\odot,{ }^{1}$ J. Benlliure, ${ }^{1}$ J. L. Rodríguez-Sánchez,${ }^{1}$ H. Alvarez-Pol, ${ }^{1}$ L. Atar, ${ }^{2}$ T. Aumann, $, 2,3,4$ V. V. Avdeichikov, ${ }^{5}$ S. Beceiro-Novo, ${ }^{1}$ D. Bemmerer ${ }^{6}$ C. A. Bertulani,${ }^{7}$ K. Boretzky, ${ }^{3}$ M. J. G. Borge, ${ }^{8}$ M. Caamaño, ${ }^{1}$ C. Caesar, ${ }^{3,2}$ E. Casarejos, ${ }^{9}$ W. Catford, ${ }^{10}$ J. Cederkall, ${ }^{5}$ M. Chartier,,${ }^{11}$ L. Chulkov, ${ }^{12}$ E. Cravo, ${ }^{13}$ R. N. P. Crespo, ${ }^{14}$ I. Dillmann, ${ }^{15,3}$ P. Díaz Fernández, ${ }^{16}$ Z. Elekes, ${ }^{17}$ J. Enders, ${ }^{2}$ O. Ershova ${ }^{3}$ A. Estrade, ${ }^{18}$ F. Farinon,,${ }^{3}$ L. M. Fraile, ${ }^{19}$ M. Freer,${ }^{20}$ D. Galaviz Redondo, ${ }^{21}$ H. Geissel, ${ }^{3}$ R. Gernhäuser ${ }^{22}$ P. Golubev,${ }^{5}$ K. Göbel, ${ }^{23}$ J. Hagdahl, ${ }^{24}$ T. Heftrich,${ }^{23}$ M. Heill, ${ }^{3}$ M. Heine, ${ }^{25}$ A. Heinz, ${ }^{24}$ A. Henriques, ${ }^{21}$ M. Holl, ${ }^{2}$ A. Hufnagel, ${ }^{2}$ A. Ignatov, ${ }^{2}$ H. T. Johansson, ${ }^{24}$ B. Jonson, ${ }^{24}$ J. Kahlbow, ${ }^{2}$ N. Kalantar-Nayestanaki, ${ }^{26}$ R. Kanungo, ${ }^{27,28}$ A. Kelic-Heil, ${ }^{3}$ A. Knyazev, ${ }^{5}$ T. Kröll, ${ }^{2}$ N. Kurz, ${ }^{3}$ M. Labiche, ${ }^{29}$ C. Langer, ${ }^{23}$ T. Le Bleis, ${ }^{22}$ R. Lemmon, ${ }^{29}$ S. Lindberg, ${ }^{24}$ J. F. D. C. Machado, ${ }^{21}$ J. Marganiec, ${ }^{2,3,30}$ A. Movsesyan, ${ }^{2}$ E. Nacher, ${ }^{8, *}$ M. A. Najafi, ${ }^{31}$ T. Nilsson, ${ }^{24}$ C. Nociforo, ${ }^{3}$ V. Panin, ${ }^{2}$ S. Paschalis, ${ }^{32}$ A. Perea, ${ }^{8}$ M. Petri, ${ }^{32}$ S. Pietri, ${ }^{3}$ R. Plag, ${ }^{3}$ R. Reifarth,${ }^{23}$ G. Ribeiro, ${ }^{8}$ C. Rigollet, ${ }^{31}$ D. M. Rossi, ${ }^{2,3}$ M. Röder, ${ }^{6,33}$ D. Savran,${ }^{30}$ H. Scheit, ${ }^{2}$ H. Simon, ${ }^{3}$ O. Sorlin, ${ }^{34}$ I. J. Syndikus, ${ }^{2}$ J. T. Taylor, ${ }^{11}$ O. Tengblad ${ }^{8}$ R. Thies, ${ }^{24}$ Y. Togano, ${ }^{35}$ M. Vandebrouck, ${ }^{34}$ P. J. F. Velho, ${ }^{21}$ V. Volkov, ${ }^{12}$ A. Wagner, ${ }^{6}$ F. Wamers, ${ }^{3,2}$ H. Weick, ${ }^{3}$ C. Wheldon, ${ }^{20}$ G. L. Wilson, ${ }^{10}$ J. S. Winfield, ${ }^{3}$ P. Woods,${ }^{18}$ D. Yakorev, ${ }^{6}$ M. Zhukov, ${ }^{24}$ A. Zilges, ${ }^{36}$ and K. Zuber ${ }^{33}$

$\left(\mathrm{R}^{3} \mathrm{~B}\right.$ Collaboration)

${ }^{1}$ IGFAE, Universidade de Santiago de Compostela, E-15782 Santiago de Compostela, Spain

${ }^{2}$ Technische Universität Darmstadt, Fachbereich Physik, 64289 Darmstadt, Germany ${ }^{3}$ GSI Helmholtzzentrum für Schwerionenforschung GmbH, Planckstraße 1, D-64291 Darmstadt, Germany

${ }^{4}$ Helmholtz, Forschungsakademie Hessen für FAIR, 64289 Darmstadt, Germany

${ }^{5}$ Lund University, Lund, Sweden

${ }^{6}$ Helmholtz-Zentrum Dresden-Rossendorf, Institute of Radiation Physics, P.O. Box 510119, 01314 Dresden, Germany

${ }^{7}$ Texas A\&M University-Commerce, Commerce, Texas 75428, USA

${ }^{8}$ Instituto de Estructura de la Materia, CSIC, E-28006 Madrid, Spain

${ }^{9}$ CINTECX, Universidade de Vigo, Vigo, Spain

${ }^{10}$ University of Surrey, Surrey GU2 7XH, England, United Kingdom

${ }^{11}$ University of Liverpool, Liverpool L69 3BX, England, United Kingdom

${ }^{12}$ NRC Kurchatov Institute, Ploshchad' Akademika Kurchatova, Moscow, Russia

${ }^{13}$ Faculdade de Sciencias, University of Lisbon, Lisboa, Portugal

${ }^{14}$ Instituto Superior Tecnico, University of Lisbon, Lisboa, Portugal

${ }^{15}$ Justus-Liebig-Universität Gießen, Gießen, Germany

${ }^{16}$ Universidad de Santiago de Compostela, E-15782 Santiago de Compostela, Spain

${ }^{17}$ ATOMKI Debrecen, Bem tér 18/c, 4026 Debrecen, Hungary

${ }^{18}$ University of Edinburgh, Edinburgh EH8 9YL, Scotland, United Kingdom

${ }^{19}$ Grupo de Física Nuclear and IPARCOS, Universidad Complutense de Madrid, CEI Moncloa, E-28040 Madrid, Spain

${ }^{20}$ University of Birmingham, Birmingham B15 2TT, England, United Kingdom

${ }^{21}$ Nuclear Physics Center, University of Lisbon, Lisboa, Portugal

${ }^{22}$ Technische Universität München, James-Franck-Strasse 1, 85748 Garching, Germany

${ }^{23}$ Johann Wolfgang Goethe-Universität Frankfurt, Max-von-Laue Strasse 1, 60438 Frankfurt am Main, Germany

${ }^{24}$ Department of Physics, Chalmers University of Technology, Fysikgränd 3, 41296 Gothenburg, Sweden

${ }^{25}$ IPHC, Strasbourg, France

${ }^{26}$ ESRIG, University of Groningen, Groningen, Netherlands

${ }^{27}$ Saint Mary's University, 923 Robie Street, Halifax, Nova Scotia, Canada B3H 3C3

${ }^{28}$ TRIUMF, 4004 Wesbrook Mall, Vancouver, British Columbia, Canada

${ }^{29}$ Science and Technology Facilities Council, Daresbury Laboratory, Warrington WA4 4AD, England, United Kingdom

${ }^{30}$ Extreme Matter Institute, Darmstadt, Germany

${ }^{31}$ KVI-CART, University of Groningen, Groningen, Netherlands

${ }^{32}$ Department of Physics, University of York, York YO10 5DD, England, United Kingdom

${ }^{33}$ Technische Universität Dresden, Institut für Kern und Teilchenphysik, Zellescher Weg 19, 01069 Dresden, Germany

*Present address: Instituto de Física Corpuscular, CSIC-Universitat de Valencia, 46071 Valencia, Spain.

Published by the American Physical Society under the terms of the Creative Commons Attribution 4.0 International license. Further distribution of this work must maintain attribution to the author(s) and the published article's title, journal citation, and DOI. 


\author{
${ }^{34}$ GANIL, Bd Henri Becquerel, 14076 Caen, France \\ ${ }^{35}$ RIKEN, Nishina Center for Accelerator-Based Science, 2-1 Hirosawa, 351-0198 Wako, Saitama, Japan \\ ${ }^{36}$ Universität zu Köln, Institut für Kernphysik, Zülpicher Straße 77, 50937 Köln, Germany
}

(Received 29 June 2021; accepted 16 November 2021; published 14 January 2022; corrected 6 April 2022)

\begin{abstract}
We measured 135 cross sections of residual nuclei produced in fragmentation reactions of ${ }^{12} \mathrm{C},{ }^{14} \mathrm{~N}$, and ${ }^{13-16,20,22} \mathrm{O}$ projectiles impinging on a carbon target at kinetic energies of near $400 \mathrm{~A} \mathrm{MeV}$, most of them for the first time, with the $\mathrm{R}^{3} \mathrm{~B} / \mathrm{LAND}$ setup at the GSI facility in Darmstadt (Germany). The use of this state-of-the-art experimental setup in combination with the inverse kinematics technique gave the full identification in atomic and mass numbers of fragmentation residues with a high precision. The cross sections of these residues were determined with uncertainties below $20 \%$ for most of the cases. These data are compared to other previous measurements with stable isotopes and are also used to benchmark different model calculations.
\end{abstract}

DOI: 10.1103/PhysRevC.105.014611

\section{INTRODUCTION}

Fragmentation reactions are widely used to investigate the structure and dynamics of the atomic nucleus [1]. Moreover, the wide range in temperature, density, and isospin asymmetry covered by participant and spectator remnants produced in these reactions offers many possibilities to explore nuclear matter under extreme conditions. Examples are the use of these reactions to constrain the equation of state of nuclear matter at supersaturation densities [2], to investigate the liquid-gas phase transition in nuclear matter [3], or to produce nuclei far from stability [4] and hypernuclei [5]. Finally, the precise understanding of these reactions has also become of importance for societal applications such as hadron therapy or space exploration [6].

Today, the effectiveness of charged particle therapy against cancer, in particular using light ions, is well established. However, the fragmentation of those ions influences the deposited depth dose and lateral profiles, eventually inducing secondary malignancies, mostly on pediatric patients [7]. Radiation protection against cosmic radiation is probably the main bottleneck for human space exploration. In this case, the fragmentation process contributes to minimize this concern because of the reduced radiobiological effectiveness of the light fragmentation products [8]. Together with the spatial and momentum distributions, the isotopic nature of the residual nuclei produced in these reactions is of utmost importance to determine the linear energy transfer in both applications. However, only few measurements with light projectiles are available and many of them do not cover the energy range of interest. The most relevant ones were obtained at Lawrence Berkeley Laboratory (LBL) $[9,10]$ and at Heavy Ion Medical Accelerator in Chiba (HIMAC) [11]. In other cases, only charge changing cross sections were measured [12-16].

In most cases, investigations based on fragmentation reactions require information on the yields of the final residual nuclei produced in those reactions. Starting with the pioneering work at the LBL [17], other heavyion facilities worldwide (e.g., Grand Accélérateur National d'Ions Lourds (GANIL), Helmholtzzentrum für Schwerionenforschung (GSI), National Superconducting Cyclotron Laboratory (NSCL), Nishina Center for Accelerator-Based Science (RIKEN), Texas A\&M University (TAMU), and Is- tituto Nazionale di Fisica Nucleare - Laboratori Nazionali del Sud (INFN-LNS) have contributed to the investigation of these reactions. Most of the reactions where the final production yields of fragmentation residues have been investigated concern medium-mass [18-20] and heavy [21-23] stable projectiles. More recently, fragmentation reactions induced by exotic projectiles have also been investigated [24,25].

Extremely peripheral reactions induced by light stable and unstable projectiles, where mostly one or few nucleons are removed, have been extensively used as spectroscopic tools to investigate the structure of these nuclei [26]. As previously mentioned, systematic studies of the residual fragments produced with light projectiles are, however, scarce, despite the increasing interest in these data because of their impact in radiotherapy and space exploration.

From a theoretical perspective, peripheral collisions of heavy ions at energies above the Fermi energy and up to few $\mathrm{GeV}$ per nucleon are well understood as a two-stage reaction mechanism [27]. In this framework the participant-spectator picture and the impulse approximation are used to describe the first stage of the collision, while statistical models provide a framework for the deexcitation of the unbound remnants (see, e.g., Refs. [28,29]). The large set of data obtained in mediummass and heavy projectile induced fragmentation reactions made it possible to benchmark and to improve the predictive power of these model calculations (see, e.g., Refs. [20,21,3035]).

The situation with light nuclei is a bit more complicated. On one hand single-nucleon removal processes are very much dependent on the structural properties of the involved nuclei [26]. The excitation energy gained by the removal of few nucleons is on average sufficient to reach the temperature threshold $(\approx 3-4 \mathrm{MeV})$ for the complete multifragmentation of the nuclei [36-38]. Therefore, models describing the residual nuclei produced in the fragmentation of light projectiles should provide an accurate description of the structural properties of those nuclei, in particular binding energies and radial distributions of protons and neutrons, and the final distribution of fragments produced in the breakup. The theoretical complexity together with the limited amount of existing data explain the present limited predictive power of model calculations describing the fragmentation process of light projectiles. 


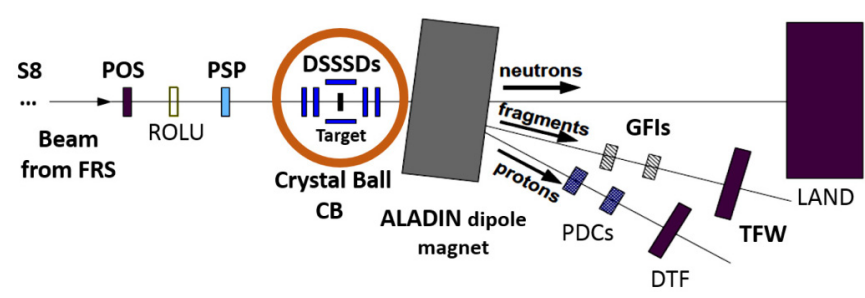

FIG. 1. Schematic view of the $\mathrm{R}^{3} \mathrm{~B} / \mathrm{LAND}$ setup in a "bird view" including the detectors used for the identification of the incoming secondary beams, the target area, the ALADIN dipole magnet, and the detectors downstream of the magnet used for the final identification of the reaction fragments.

In this paper we present a rather complete set of production cross sections of isotopically separated residual nuclei produced in fragmentation reactions induced by ${ }^{12} \mathrm{C},{ }^{14} \mathrm{~N}$, and several oxygen isotopes covering a large range in neutron excess with typical energies near $400 \mathrm{~A} \mathrm{MeV}$, impinging on a carbon target. The measurements were done with the $\mathrm{R}^{3} \mathrm{~B} / \mathrm{LAND}$ setup [39], providing at the same time large acceptance for most of the produced fragments and their unambiguous isotopic identification from the determination of their magnetic rigidity, energy loss, and time of flight. These measurements have been used to investigate single-particle state occupancy by taking advantage of quasifree scattering reactions in inverse kinematics [40-52].

The paper is organized as follows. The experimental method and the data sorting procedure are described in Sec. II. In Sec. III we present the measured cross sections compared with existing data and we use the new data to benchmark stateof-the-art model calculations. Finally, in Sec. IV we summarize the main results and present the conclusions of this paper.

\section{EXPERIMENT AND DATA SORTING}

\section{A. Experimental setup}

The experiment was performed with the $\mathrm{R}^{3} \mathrm{~B} / \mathrm{LAND}$ setup at the GSI accelerator facilities in Darmstadt (Germany). A primary beam of ${ }^{40} \mathrm{Ar}$ was accelerated with the UNILAC and SIS18 accelerators up to an energy near 500A MeV. Then, the beam was guided to the FRagment Separator (FRS) [53], where secondary radioactive beams were produced by fragmentation reactions on a thick beryllium production target $\left(4.011 \mathrm{~g} / \mathrm{cm}^{2}\right)$.

Secondary beams, separated by the FRS according to their mass-over-charge ratio, were transported to the $R^{3} B / L A N D$ experimental hall. In order to produce beams of light elements, covering large isotopic chains, different magnetic settings of the FRS centered around nominal mass-to-charge ratios ranging from 1.5 to 3 were used. The secondary beams, with a Gaussian profile of $1.1 \times 0.8 \mathrm{~cm}^{2}$ full width at half maximum, impinged on a target wheel hosting several carbon targets with thicknesses of $0.94,0.561$, and $0.416 \mathrm{~g} / \mathrm{cm}^{2}$, where fragmentation reactions were induced.

The $\mathrm{R}^{3} \mathrm{~B} / \mathrm{LAND}$ setup, shown in Fig. 1, was designed to perform complete kinematic studies of nuclear reactions induced by relativistic radioactive beams [39]. The ions in the incoming cocktail beam were identified on an event-by-event basis. The atomic number was obtained from the energy loss of the ions in position-sensitive PIN silicon diodes (PSPs). The mass-over-charge ratio was deduced from their velocity and trajectory determined by time-of-flight and position measurements using plastic scintillators, located at the final focal plane of the FRS (S8), and a position-sensitive scintillator (POS), located at the entrance of the experimental hall. A veto scintillator system (ROLU) was used to center the beam on target and to reject the halo in the beam.

In front and behind of the target, two pairs of doublesided silicon-strip detectors (DSSSDs), provided additional measurements of the energy loss and position of the incoming projectiles and outgoing fragments. Finally, the crystal ball (CB) calorimeter [54], consisting of a spherical array of 159 $\mathrm{NaI}$ crystals, covering a solid angle of $\approx 90 \%$ of $4 \pi$, with a 2-cm-diameter aperture at the entrance, surrounded the target area. This detector was equipped for the forward angles with crystals with dual readout electronics, enabling the simultaneous detection of photons and light-charged particles. For the particular results presented in this paper, the signals from this detector were only used as trigger.

Charged reaction fragments were bent by a largeacceptance dipole magnet (ALADIN) and identified in different detection arms behind the magnet. The so-called fragment arm provided the identification of the reaction fragments. Energy loss and time signals were measured by a plastic scintillator wall (TFW), whereas the position hits were recorded using large fiber detectors (GFIs) [55] and also TFWs. These measurements, together with the DSSSD signals, allowed us to reconstruct the trajectories of the fragments through the dipole magnet. A dedicated trajectory reconstruction algorithm based on iterative calculations to minimize the parameters of fragment paths $[40,43]$ provided the momentum and isotopic identification for all fragments. Additionally, the proton arm provided the identification of light-charged particles through energy loss and position measurements $[41,42,50]$. Neutrons, not affected by the magnetic field, were detected with the neutron detector LAND [56]. In the present paper the identification of light-charged particles and neutrons was not used.

\section{B. Data sorting}

Figure 2 depicts an example of a secondary cocktail beam identification matrix for a magnetic setting of the FRS centered around $A / Z \sim 2.1$ nuclei. The atomic number was obtained from the energy loss measurements provided by the PSP and DSSSD detectors located upstream of the target. The mass-over-charge identification was achieved from the timeof-flight and position measurements provided by the plastic scintillators located at S8 and the entrance of the experimental cave (POS). A double condition on the atomic number identification obtained from the energy loss measurements in the PSP and DSSSD detectors was implemented to improve the selection of the projectile nucleus.

The isotopic identification of the fragments produced at the target was done following a three-step process: a selection of the incoming secondary projectile, a double energy loss identification in the DSSSD detector downstream of the target and the TFW detector, and the mass-to-charge ratio from the 


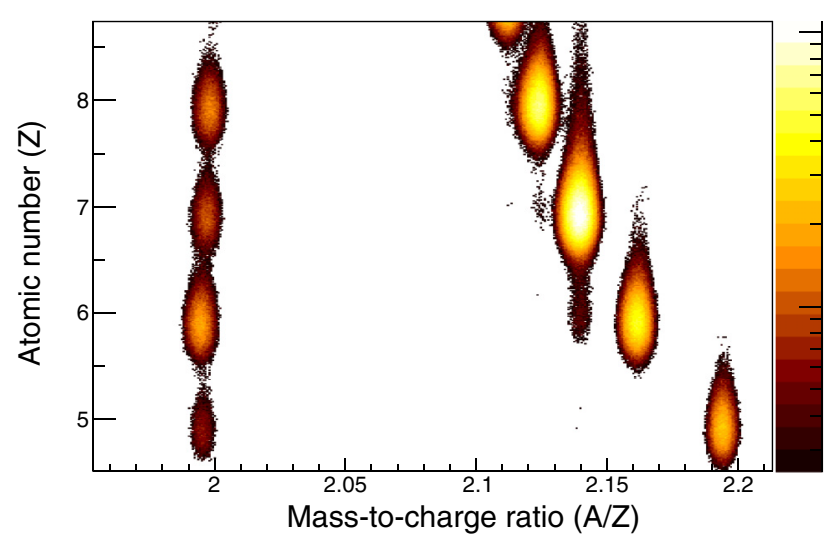

FIG. 2. Projectile identification matrix for beams centered around $A / Z=2.1$.

reconstructed trajectories and measured time of flight. The trajectories were obtained from the positions of all fragments at the DSSSD detectors placed downstream of the target, and from the GFIs and the TFW detectors behind ALADIN. Their time of flight was obtained from the signals provided by the POS and TFW scintillators. Figure 3 depicts the isotopic identification of the fragments produced in the interaction of ${ }^{14} \mathrm{O}$ projectiles with a carbon target.

\section{Fragmentation cross sections}

The cross sections of the fragments produced in the interactions of several oxygen isotopes $\left({ }^{13,14,15,16,20,22} \mathrm{O}\right)$, together with ${ }^{14} \mathrm{~N}$ and ${ }^{12} \mathrm{C}$, impinging on a carbon target were obtained by normalizing the number of identified fragmentation residues to the number of incoming projectiles and the number of scattering centers per surface unit in the carbon target. Correction factors taking into account the experimental geometrical acceptance, the probability for secondary reactions, and the efficiency of the acquisition trigger were applied and are described in the following paragraphs.

In this experiment we used two different acquisition triggers. The fragment trigger required the spill-on logical signal

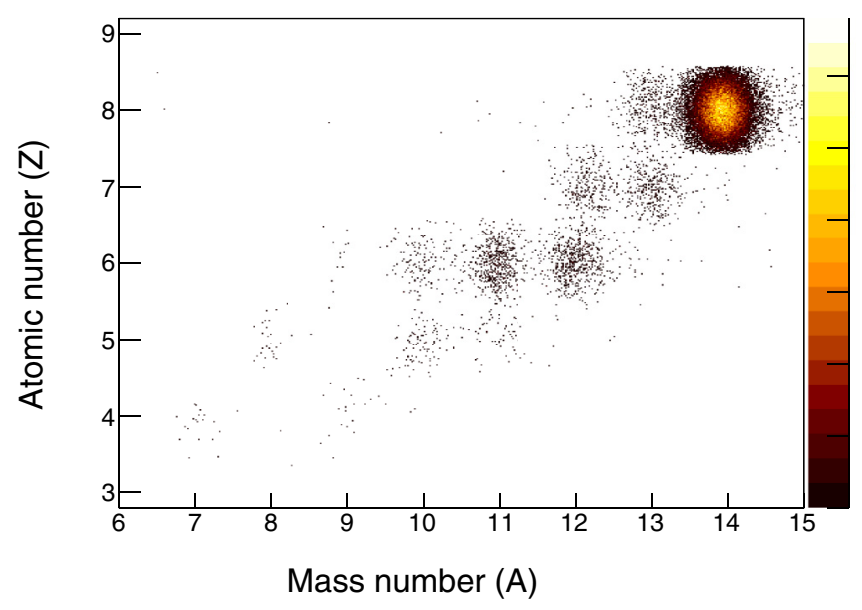

FIG. 3. Identification matrix of the fragments produced in reactions induced by ${ }^{14} \mathrm{O}$ projectiles impinging on a carbon target.

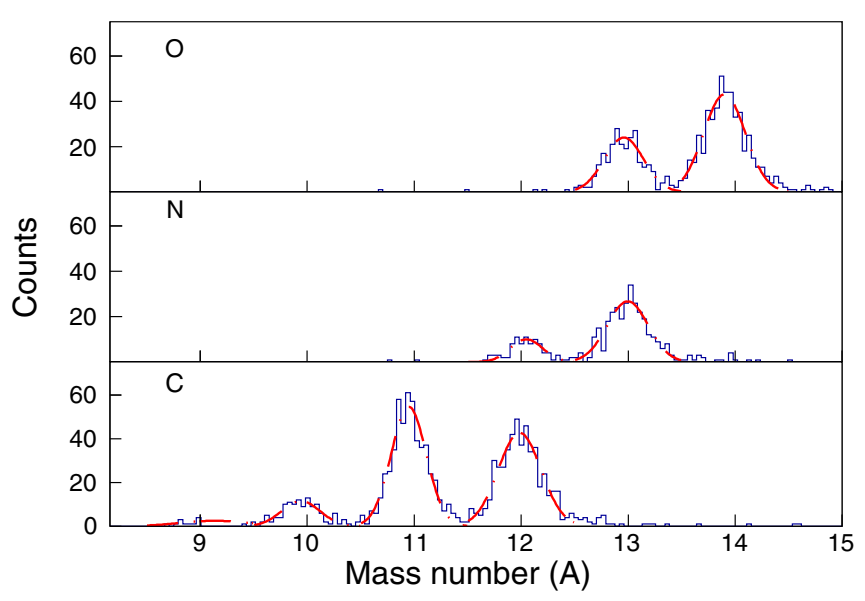

FIG. 4. Isotopic identification of oxygen, nitrogen, and carbon fragments produced in reactions induced by ${ }^{14} \mathrm{O}$ projectiles impinging on a carbon target.

from the accelerator, a signal in the POS, and TWF detectors in anticoincidence with the ROLU veto. Ideally, this trigger could be used to determine both projectile and fragment fluxes using the counts registered by the last detector of the setup (TFW). However, fragments produced in single-nucleon removal reaction channels had a strong contribution of the unreacted beam that prevented the precise determination of its production yield. Moreover, because of the large number of incoming projectiles this trigger had to be downscaled.

To overcome this problem, we used a second trigger for an accurate determination of the yields of the fragmentation residues. This so-called reaction trigger, in addition to the above-mentioned conditions, also required an energy signal in the $\mathrm{CB}$ detector of at least $100 \mathrm{keV}$. This condition is understood as an indication that the incoming projectile has reacted with the target. The advantages of using this reaction trigger are evident: this trigger does not require any downscaling and the undesired projectile contributions are not registered, ensuring a proper fragment identification even for the single nucleon removal channels.

In Fig. 4 we present mass distributions of carbon, nitrogen, and oxygen fragments produced in the fragmentation of ${ }^{14} \mathrm{O}$ projectiles on a carbon target recorded with the reaction trigger. The unambiguous isotopic identification of the different fragmentation residues gave an accurate determination of their yields.

The use of two different acquisition triggers for the determination of the number of incoming projectiles and the yields of the fragmentation residues required an additional correction taking their different efficiencies into account. This correction showed a dependence with the difference in atomic and mass numbers between projectile and residual nucleus, ranging from 10 to $20 \%$ with uncertainties between 2 and $5 \%$, for most of the cases.

The geometric acceptance was obtained from a detailed GEANT4 simulation of the experiment. In this simulation realistic physics parameters, such as beam-spot size at the target, the momentum of each outgoing fragment, the ALADIN magnetic field, and the electromagnetic interactions of the in- 
coming and outgoing fragments, are considered. For a typical one proton knockout reaction, this value is roughly $100 \%$, while for cases involving a large number of nucleons removed (more than six nucleons) it can decrease to values below $50 \%$.

The correction for secondary reactions of the fragments mostly in the thick reaction targets has been evaluated using total reaction cross sections obtained with the code KAROL [57]. For fragments between oxygen and beryllium, the corresponding correction varies between 3.6 and $7.8 \%$. Finally, because we use the same detectors for counting projectiles and fragments, detection efficiencies cancel.

\section{RESULTS}

\section{A. Measured cross sections}

Using the methodology presented in the previous sections we could determine the isotopic production cross sections of residual nuclei obtained in reactions induced by ${ }^{12} \mathrm{C},{ }^{14} \mathrm{~N}$, and ${ }^{13,14,15,16,20,22} \mathrm{O}$ projectiles impinging on a carbon target at energies ranging from $350 \mathrm{~A}$ to $450 \mathrm{~A} \mathrm{MeV}$. The 135 cross sections obtained in this paper are reported in the Appendix. We measured 101 of these cross sections, mostly the ones obtained with unstable projectiles, for the first time. We also measured 34 cross sections obtained with ${ }^{12} \mathrm{C},{ }^{14} \mathrm{~N}$, and ${ }^{16} \mathrm{O}$ projectiles in previous works, at the same or similar energies. The joint evaluation of these sets of data allowed us to assess their validity. The lighter fragments measured in this paper are beryllium isotopes in reactions induced by oxygen projectiles, and lithium isotopes in reactions induced by carbon and nitrogen projectiles. The main limitations to measure lighter fragments are the reduced geometrical acceptance and statistics.

The uncertainties associated to the measured cross sections include statistical and systematic contributions. Statistical uncertainties vary between a few percent for the most produced fragments (one nucleon removal) and $15 \%$ for the lighter fragments. Systematic uncertainties are associated with the different corrections applied to the measured yields to obtain production cross sections, described in the previous section, together with the uncertainty associated to the target thickness. The main sources of systematic uncertainties are the geometrical acceptance and the trigger efficiency correction. The uncertainty associated with the geometrical acceptance is rather small, below 5\%, for the cases where the setup acceptance is large. However, it increases above $20 \%$ for the few cases where the difference between the projectile and the final residues is larger than eight mass units with a geometrical acceptance below $60 \%$. Finally, the uncertainty in the trigger efficiency correction is on average $5 \%$.

Fragmentation reactions induced by stable ${ }^{12} \mathrm{C}$ on a carbon target have been previously investigated at different energies and therefore represent a good case to benchmark the new measurements. Figure 5 depicts in three consecutive panels the production cross sections of several boron [Fig. 5(a)], beryllium [Fig. 5(b)], and lithium [Fig. 5(c)] isotopes measured in this paper (close symbols) compared to measurements reported in literature [9-11] over a broad range of energies.

The most widely measured reaction channel is the singleproton removal leading to the production of ${ }^{11} \mathrm{~B}$. As can be
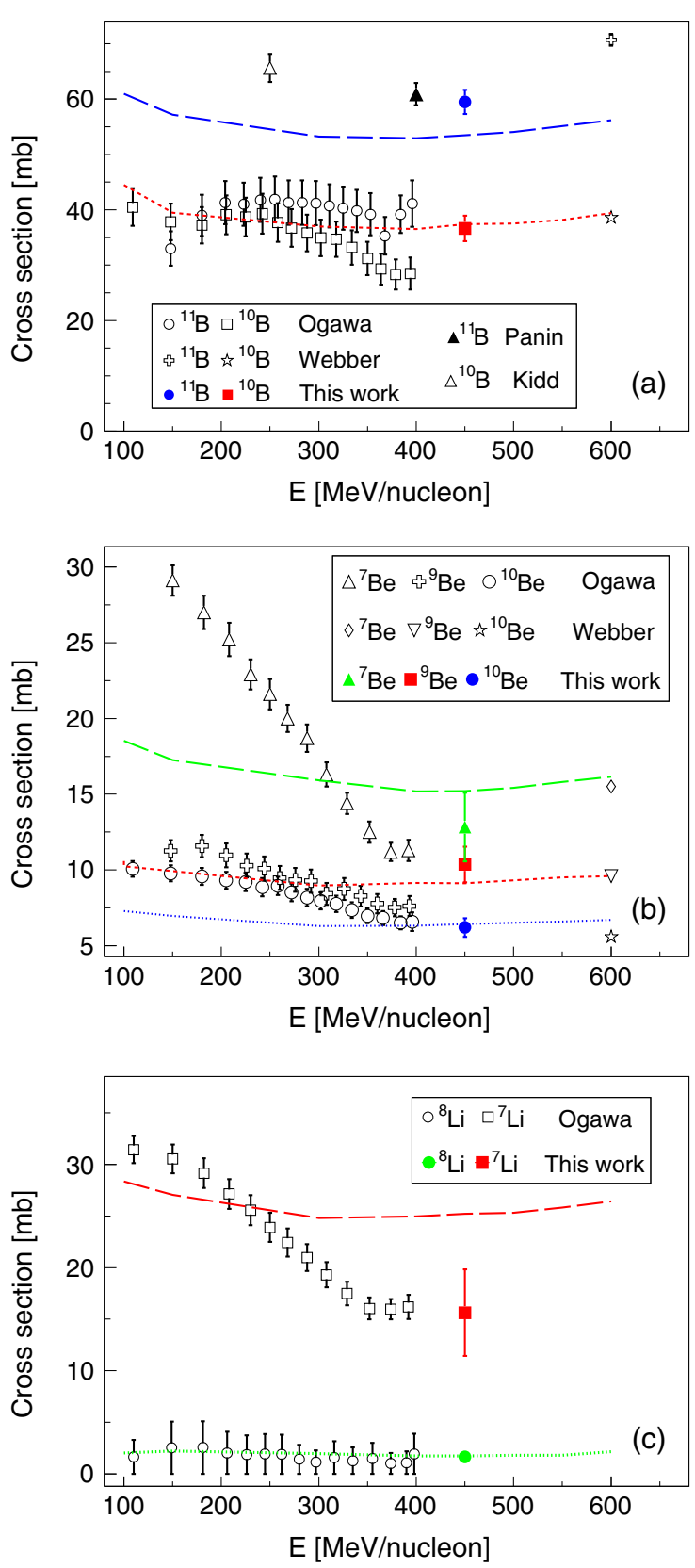

FIG. 5. Energy dependence of the production cross sections of ${ }^{10,11} \mathrm{~B}$ (upper panel), ${ }^{7,9,10} \mathrm{Be}$ (middle panel), and ${ }^{6,7} \mathrm{Li}$ (lower panel) isotopes produced in reactions induced by ${ }^{12} \mathrm{C}$ projectiles impinging on a carbon target at energies between $100 \mathrm{~A}$ and $600 \mathrm{~A} \mathrm{MeV}$. The different symbols represent measurements obtained in this paper and also found in the literature. The lines represent model predictions obtained with the code ABRABLA07 (see text for details).

seen in Fig. 5(a), the value obtained in this paper (solid dot) is in very good agreement $(\approx 0.4 \sigma)$ with the recent result of Panin et al. (full triangle) [58], and reasonable agreement $(\approx 1.8 \sigma)$ with Kidd et al. (empty triangle) [9], although this measurement was obtained at a slightly lower energy $(250 \mathrm{~A} \mathrm{MeV})$. The difference with Webber et al. (empty cross) [10] is clearly larger $(\approx 4.6 \sigma)$, but this could be explained by the larger energy $(600 \mathrm{~A} \mathrm{MeV})$. 


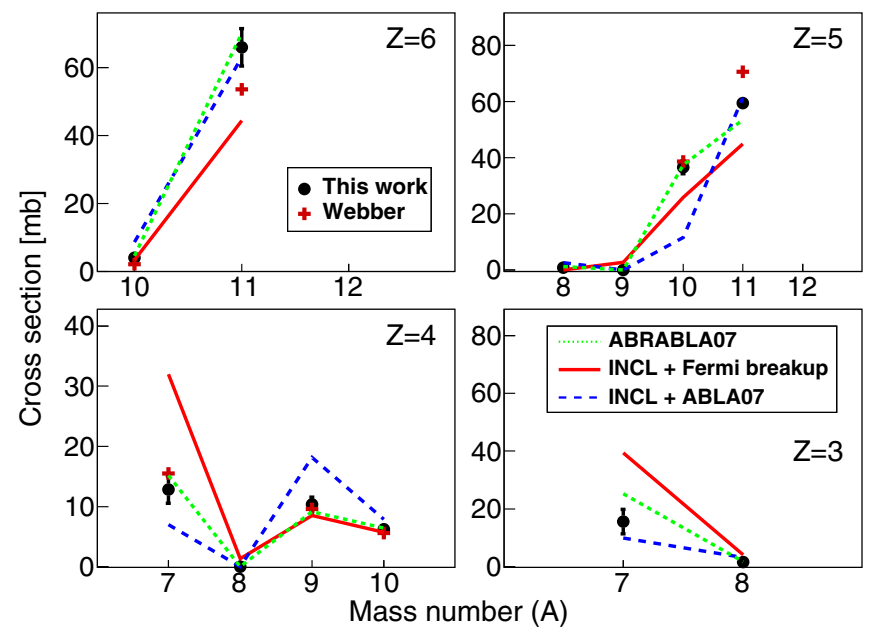

FIG. 6. Isotopic distributions of the cross sections of residual nuclei produced in the reaction ${ }^{12} \mathrm{C}(450 \mathrm{~A} \mathrm{MeV})+\mathrm{C}$ measured in this paper (solid points) and by Webber et al. [10] (red crosses). The lines represent predictions obtained with the reaction models ABRABLA07 (dotted lines), INCL + Fermi breakup (solid lines), and $\mathrm{INCL}+$ ABLA07 (dashed lines).

Conversely, the results obtained by Ogawa et al., (circles) [11] are $\approx 40 \%$ smaller over the same energy range. The cross section for the removal of a single proton and a single neutron producing ${ }^{10} \mathrm{~B}$ is also in good agreement with the measurement of Webber et al. The measurements of Ogawa et al. are again smaller, this time by $\approx 30 \%$.

For the beryllium isotopes [Fig. 5(b)] we observe the same tendency. Our results are in good agreement with Webber et al. [10], while the measurements of Ogawa et al. [11] are smaller, in particular for ${ }^{7} \mathrm{Be}$ and ${ }^{9} \mathrm{Be}$. For the lithium isotopes [Fig. 5(c)] a better agreement is observed for the cross sections obtained by Ogawa et al., and ours. Hence, we can conclude that the cross sections we have obtained for ${ }^{12} \mathrm{C}$ projectiles are in good agreement with most of the previously published cross sections, except for the ones obtained by Ogawa et al., which are systematically smaller.

Figures 6-8 display the isotopic distributions of all cross sections obtained in this paper. In these figures we also report the cross sections of residual fragments produced in reactions induced by ${ }^{12} \mathrm{C},{ }^{14} \mathrm{~N}$, and ${ }^{16} \mathrm{O}$ projectiles obtained by Webber et al. [10] in inverse kinematics at $600 \mathrm{~A} \mathrm{MeV}$ (red crosses). Also in this case we find a good agreement with the previous measurements. We only observe that our cross sections are slightly smaller, on average $\approx 10 \%$, but this difference could be explained by the different energy used in both measurements.

The isotopic distributions of the cross sections shown in these figures follow, in general, the expected trend with a clear decrease in the cross sections with the difference in mass number between the projectile nucleus and the final fragment. However, because of the large isotopic range in the final fragments covered with the different projectiles, we also observe some structural effects in these isotopic distributions. A clear example is the absence of the unbound ${ }^{9} \mathrm{~B}$ and ${ }^{8} \mathrm{Be}$ in the isotopic distribution of residual cross sections obtained with

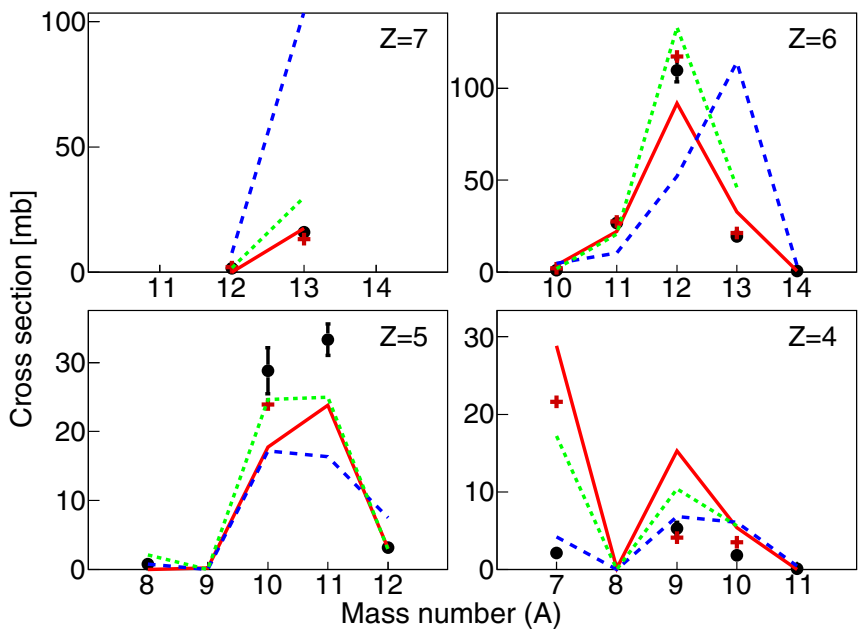

FIG. 7. Isotopic distributions of the cross sections of residual nuclei produced in the reaction ${ }^{14} \mathrm{~N}(450 \mathrm{~A} \mathrm{MeV})+\mathrm{C}$ measured in this paper (solid points) and by Webber et al. [10] (red crosses). The lines represent predictions obtained with the reaction models ABRABLA07 (dotted lines), INCL + Fermi breakup (solid lines), and INCL + ABLA07 (dashed lines).

neutron-deficient ${ }^{13,14,15} \mathrm{O}$ (Fig. 8) and stable ${ }^{12} \mathrm{C}$ (Fig. 6), ${ }^{14} \mathrm{~N}$ (Fig. 7), and ${ }^{16} \mathrm{O}$ (Fig. 8) projectiles. A cluster effect is also noticed by the dominant production of ${ }^{12} \mathrm{C}$ in reactions induced by ${ }^{16} \mathrm{O}$ projectiles (Fig. 8), although a fraction of its initial production is lost through the triple-alpha decay [59].

We also observe clear structural effects in the isotopic distributions of the residual cross sections obtained with neutron-rich ${ }^{20,22} \mathrm{O}$ (Fig. 8) projectiles. In these cases, the strong even-odd staggering in the cross sections for oxygen and nitrogen residual nuclei clearly follows the evolution of the neutron-separation energies. A clear example is the low cross section for the production of ${ }^{15} \mathrm{C}$, also caused by the low value of the corresponding neutron-separation energy.

Another interesting issue concerns the observed production of ${ }^{14} \mathrm{C}$ in reactions induced by ${ }^{14} \mathrm{~N}$ projectiles, and ${ }^{12} \mathrm{C}$ and ${ }^{11} \mathrm{~B}$ in reactions induced by ${ }^{13} \mathrm{O}$. Those isotopes are expected not to be produced in fragmentation reactions but rather in charge-exchange reactions where Gamow-Teller or inelastic processes may contribute $[60,61]$.

\section{B. Fragmentation models}

Fragmentation reactions are usually understood as a two step process. The first stage corresponds to the fast interaction between projectile and target nuclei, that according to the Glauber model [62] can be described in terms of individual nucleon-nucleon collisions, leading to a participant region at midrapidity, and two unbound remnants of projectile and target nuclei. During a second stage of the reaction, projectile and target remnants are expected to thermalize and evolve towards a final residue by losing the excess of energy and angular momentum by emitting gamma rays, nucleons, or light clusters. This second stage is usually modeled following the statistical evaporation model. 


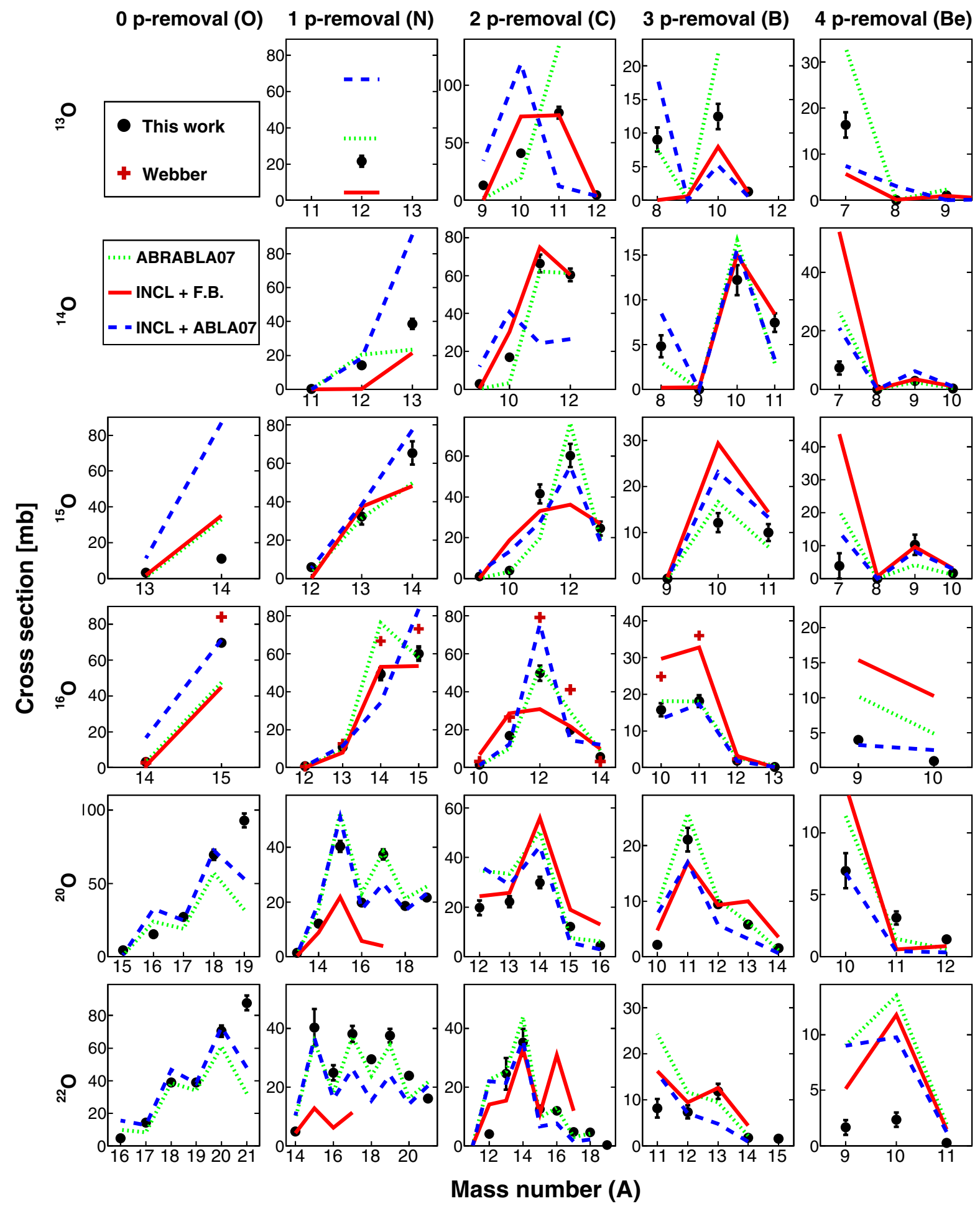

FIG. 8. Isotopic distributions of the cross sections of residual nuclei produced in reactions induced by ${ }^{13} \mathrm{O},{ }^{14} \mathrm{O},{ }^{15} \mathrm{O},{ }^{16} \mathrm{O},{ }^{20} \mathrm{O}$, and ${ }^{22} \mathrm{O}$ impinging on a carbon target. Predictions obtained with the codes ABRABLA07 (dotted lines), INCL + Fermi breakup (solid lines), and INCL + ABLA07 (dashed lines) are also shown.

In this paper we propose to take advantage of the large data set obtained to benchmark reaction models, that are generally used to model the fragmentation of medium-mass and heavy nuclei. In particular we will use two different approaches of the Glauber model to describe the first stage of the collision, the abrasion code ABRA by Gaimard and Schmidt [28] and the
Liège intranuclear-cascade model INCL developed by Cugnon and collaborators [31].

The deexcitation of the projectile remnants is modeled using the statistical evaporation code ABLA07 [63], based on the Weisskopf formalism [64]. Moreover, we will also use models describing the multifragmentation process. This channel plays 
a role in reactions induced by light projectiles where relatively small values of the excitation energy gained in the reaction can be comparable with the total binding energy of the remnant. In that case nuclei breakup. In particular, we will use the multifragmentation model implemented in the ABLA07 code $[63,65]$ and the Fermi breakup model [66]. In the following paragraphs we will provide some details about these different models.

The abrasion model represents a geometrical solution of Glauber's picture justified by the adiabatic approximation. This approximation assumes that at projectile energies well above the Fermi energy only the nucleons in the overlap region between projectile and target nuclei (participants) interact strongly, while the nucleons outside this zone (spectators) remain undisturbed. Masses of the remaining nuclei (projectile and target spectators) can thus be obtained from the geometrical overlap between projectile and target nuclei as a function of the impact parameter.

In the ABRA formulation of this model, the neutron-toproton $(N / Z)$ ratio of the remaining nucleus is determined just by statistical considerations, where the distribution of the $N / Z$ ratio after the collision is calculated by using the hypergeometrical approach [67]. Thus, the average $N / Z$ ratio of the remaining nucleus corresponds to that of the initial nucleus, although with large statistical fluctuations, which lead to large variations in the $N / Z$ ratio of the reaction products [21].

In ABRA, the excitation energy gained by the spectators is computed from particle-hole excitations assuming a Fermigas distribution of the nucleons and an isospin independent potential with a depth of $47.4 \mathrm{MeV}$. This simple picture generates a linear distribution for the excitation energy gained in a single-nucleon removal between 0 and $40 \mathrm{MeV}$. To obtain the excitation function for the removal of " $n$ " nucleons the model just convolutes the single-nucleon excitation function $n$ times. In this approximation the mean excitation energy gained by the spectators by the removed nucleon during the fragmentation stage is $13.5 \mathrm{MeV}$. Previous investigations indicate that calculations based in this model provide reasonable results for reactions induced by light projectiles [44]. However, in fragmentation reactions induced by medium-mass and heavy nuclei measured production cross sections of residual nuclei can only be described reasonably well when increasing the excitation energy by a factor $2[21,30]$. The role of realistic density profiles for neutrons and protons, the use of an isospin independent potential, and the role of final-state interactions might explain this phenomenological increase of the excitation energy.

The second model that we have used to describe projectile fragmentation reactions is the dynamic intranuclear cascade model, in particular the INCL code [31], which can be considered as a Monte Carlo method to solve numerically the dynamic transport equations describing particle-nucleus and nucleus-nucleus collisions. The nature of INCL is essentially classical, assuming that hadrons are perfectly localized in phase space and that they are bound by a potential. In this approach, the nuclear collision is treated as successive relativistic binary hadron-hadron collisions separated in time, where the positions and momenta of hadrons are followed as time evolves. It is also assumed that hadrons move along straight trajectories until they undergo a collision with another hadron or until they reach the surface, where they can eventually escape. Cross sections are determined from a set of collision events taken at different impact parameters for which nucleon positions and momenta are initially sampled for each participant nucleus. Here, one must note that projectile and target nuclei are treated in different ways. On the one hand, assuming reactions in inverse kinematics, the projectile nucleus is represented by a potential well according to the Woods-Saxon distribution, the radius of which depends on the nucleon momentum [29]. The potential well depth for nucleons is calculated according to optical models, including isospin dependencies [68], while radii and diffuseness parameters of the neutron and proton density profiles are taken from Hartree-Fock-Bogoliubov calculations since they provide a good description of single-nucleon removal processes in spallation reactions [33]. In the extended version of INCL considering composite target nuclei $(A<18)$, the density profiles are described by modified-harmonic oscillator distributions and the nucleon momenta are described by Gaussian distributions considering the same rms momentum $\left(3 / 5 p_{F}\right)$ for protons and neutrons, with $p_{F}=270 \mathrm{MeV} / c$ (see Ref. [31] for more details).

For the dynamic description of the collision between projectile and target nuclei, each event has a given impact parameter $b$, ranging from zero to a value $b_{\max }$ given by the sum of the maximum radius of projectile and target nuclei. If two hadrons approach each other closer than a maximum distance, they interact. The minimum distance is calculated from energy-dependent parametrizations of the hadron-hadron interaction cross sections for all possible collisions according to Ref. [69]. During the cascade process, the particles inside the projectile volume are divided into participants and spectators. Participants are defined as particles that have collided with at least one other participant, while the remaining particles are spectators. Collisions between spectators are forbidden in order to eliminate the spontaneous boiling of the Fermi sea. For projectile nucleons, a strict Pauli blocking is also applied to the first collision to account for surface effects and for effects of the depletion of the Fermi sea [70], while it is neglected for target nucleons [31]. For the subsequent collisions, INCL applies the Pauli principle according to the usual procedures by means of statistical blocking factors. In addition, a consistent dynamical Pauli blocking is applied to all particles at the end of the cascade process to reject nonphysical results (see Ref. [29] for more details).

The code ABLA07 describes the deexcitation of the hot excited remnants following Weisskopf's formalism [64]. This code includes the possibility for the emission of $\gamma$ rays, neutrons, light-charged particles, intermediate-mass fragments, and fission [71,72]. For a more realistic description of the deexcitation process, the separation energies and the emission barriers for charged particles are also considered according to the atomic mass evaluation from 2016 [73] and the Bass potential [74], respectively.

Moreover, in ABLA07 remnants exceeding a threshold temperature undergo a complete breakup into fragments and nucleons. This mass-dependent freeze-out temperature is calculated according to the prescription given by Natowitz and 
collaborators [75]. The size of the clusters is sampled according to an exponential distribution with a slope parameter $\tau$, calculated assuming a linear dependence on the excitation energy per nucleon in the temperature regime of interest, as explained in Refs. [76,77]. The atomic number of the corresponding fragment is also sampled at the same time from a Gaussian distribution centered at a value $Z_{\text {mean }}$, determined by imposing to the clusters the same $A / Z$ value of the hot remnant. The width of this distribution is obtained as the ratio between the freeze-out temperature and the symmetry term of the nuclear equation of state [78]. The excitation energies of the resulting hot residues are determined by assuming thermal equilibrium at the freeze-out temperature [65]. Finally, each of the breakup residues with a mass number greater than $A=4$ will then enter the statistical deexcitation stage.

The second approach we have used to describe the breakup channels is based on the Fermi breakup model [66]. This is a pure statistical model where the weight of each breakup channel containing $n$ fragments with masses $m_{i}(i=1, \ldots, n)$ in a volume $V_{f}$ is calculated in the microcanonical approximation as

$$
\begin{aligned}
\Gamma_{f}\left(E_{\mathrm{kin}}, n\right)= & \frac{S_{n}}{G_{n}}\left(\frac{V_{f}}{(2 \pi \hbar)^{3}}\right)^{n-1}\left(\frac{\prod_{i=1}^{n} m_{i}}{m_{0}}\right)^{3 / 2} \\
& \times \frac{(2 \pi)^{3(n-1) / 2}}{\Gamma(3(n-1) / 2)}\left(E_{\mathrm{kin}}-U_{f}\right)^{(3 n-5) / 2},
\end{aligned}
$$

where $U_{f}$ is the Coulomb barrier, $m_{0}=\sum_{i=1}^{n} m_{i}$ is the mass of the decaying nucleus, $S_{n}=\prod_{i=1}^{n}\left(2 S_{i}+1\right)$ is the spin degeneracy factor ( $S_{i}$ is the $i$ th particle spin), and $G_{n}=\prod_{j=1}^{k} n_{j}$ ! is the particle identity factor $\left(n_{j}\right.$ is the number of particles of kind $j) . E_{\text {kin }}$ is the total kinetic energy of particles at infinity that is related to the prefragment excitation energy $\left(E^{*}\right)$ as

$$
E_{\mathrm{kin}}=E^{*}+m_{0} c^{2}-\sum_{i=1}^{n} m_{i} c^{2} .
$$

In this model all final-state fragments are assumed to be bound and therefore no further deexcitation is required.

\section{Benchmark of model calculations}

The results of these model calculations are shown in Figs. 5-8. In Fig. 5 the different lines depict predictions obtained with the ABRABLA07 code. A more detailed benchmark is done in Figs. 6-8 where we report results obtained with all model calculations described in the previous section: ABRABLA07 (dotted lines), INCL coupled to the Fermi breakup (solid lines), and INCL coupled to ABLA07 (dashed lines). In general, we can conclude that the three codes provide an overall reasonable description of the measured cross with the exception of few particular residual nuclei.

A detailed analysis indicates that the ABRABLA07 code provides in general the best description of the measured data. Indeed, for most of the measured cross sections the model provides a description of the data with an accuracy better than $20 \%$. The main deviations are observed for the production of ${ }^{7} \mathrm{Be}$ in reactions induced by ${ }^{14} \mathrm{~N},{ }^{13} \mathrm{O}$, and ${ }^{14} \mathrm{O}$. On the other hand, we also observe a large difference between our mea- surement and Webber's measurement for the production cross section of ${ }^{7} \mathrm{Be}$ in reactions induced by ${ }^{14} \mathrm{~N}$ while the cross sections for all other beryllium isotopes are rather similar (see Fig. 7). This could indicate that the cross sections obtained for ${ }^{7} \mathrm{Be}$ in our measurements could be underestimated. Important discrepancies are also observed for the production of ${ }^{9} \mathrm{Be}$ and ${ }^{10} \mathrm{Be}$ in reactions induced by ${ }^{22} \mathrm{O}$ projectiles. The fact that the predictions of the three codes are rather similar could indicate an underestimation of the measured cross sections caused by an overprediction of the geometrical acceptances. Those are nuclei measured with a geometric acceptance below $60 \%$ and with relatively large uncertainties in the estimated acceptances.

We also observe a clear underestimation of the calculated one-neutron removal cross sections in reactions induced by ${ }^{20} \mathrm{O}$ and ${ }^{22} \mathrm{O}$ projectiles. Similar conclusions were previously obtained for neutron-rich heavier nuclei $[35,79]$. In principle, this could be a signature of the extended radial distribution of neutrons in these projectile nuclei, not considered in these model calculations. Another explanation would be an overestimation of the excitation energy gained by the remnants produced in these reaction channels for neutron-rich nuclei.

We also conclude that the standard version of the ABRA abrasion model provides a good description of the fragmentation data without any modification of the excitation energy calculated by default. The $40 \%$ reduction in the excitation energy proposed in Ref. [44] for the same reactions could be due to the fact that this investigation focused on very peripheral reactions while in the present paper we also include midperipheral collisions. A final interesting conclusion is that according to this model calculation the contribution of multifragmentation channels to the final cross sections is smaller than $15 \%$, indicating that the role of this reaction mechanism is smaller than initially anticipated.

Predictions obtained with the INCL model coupled to ABLA07 show a rather good description of the data. However, an important remark is that first predictions for ${ }^{20} \mathrm{O}$ and ${ }^{22} \mathrm{O}$ fragmentation obtained with the extended version of INCL describing ion-ion collisions provided a rather disappointing result. In this particular case we used calculations using the standard INCL version where protons are considered as target nuclei instead of the carbon ions used in the other calculations.

The main deviations correspond to the overprediction for the production cross section of ${ }^{7} \mathrm{Be}$ observed in many of the reactions, already discussed in the previous paragraphs. We also observe a general overprediction of single-proton removal channels in reactions induced by ${ }^{14} \mathrm{~N}$ and neutrondeficient oxygen isotopes $\left({ }^{13} \mathrm{O}\right.$ and $\left.{ }^{14} \mathrm{O}\right)$, and an underprediction of single-neutron removal channels in reactions induced by neutron-rich oxygen isotopes $\left({ }^{20} \mathrm{O}\right.$ and $\left.{ }^{22} \mathrm{O}\right)$. This result is quite surprising given that INCL includes a realistic description of the radial distributions of protons and neutrons [33]. A final interesting observation is the rather accurate description of the charge-exchange channels measured in this paper, ${ }^{14} \mathrm{C}$ in reactions induced by ${ }^{14} \mathrm{~N}$ projectiles, and ${ }^{12} \mathrm{C}$ and ${ }^{11} \mathrm{~B}$ in reactions induced by ${ }^{13} \mathrm{O}$ projectiles.

Results obtained with the INCL extended version describing ion-ion collisions, coupled to the Fermi breakup model, also show a rather good predictive power within the range 
of validity of the Fermi breakup model $(A<18)$. The main discrepancies observed are the same overprediction of the production cross section of ${ }^{7} \mathrm{Be}$ in most of the investigated reactions, and a certain overestimation of the one-neutron removal channel together with an underestimation of the one-proton removal channels. In addition, there is a clear underestimation of the production cross section of ${ }^{12} \mathrm{C}$ in reactions induced by ${ }^{15} \mathrm{O}$ and ${ }^{16} \mathrm{O}$ projectiles. This reaction channel could be influenced by a cluster effect in ${ }^{16} \mathrm{O}$. In principle this cluster effect should manifest through the values of the masses used in the calculations, but not in the description of the particular unbound states leading to cluster decays that are not considered in the deexcitation models used in this paper. Moreover, the Fermi breakup model does not use evaluated masses but calculated ones with the liquid-drop model. The values of the masses could also explain other deviations in the predictions with respect to the measured cross sections.

\section{SUMMARY AND CONCLUSIONS}

The $\mathrm{R}^{3} \mathrm{~B} / \mathrm{LAND}$ setup installed at GSI was used to measure the isotopic cross sections of residual nuclei produced in fragmentation reactions induced by stable light projectiles, such as ${ }^{12} \mathrm{C},{ }^{14} \mathrm{~N}$, and ${ }^{16} \mathrm{O}$ projectiles, but also by unstable oxygen isotopes $\left({ }^{13} \mathrm{O},{ }^{14} \mathrm{O},{ }^{15} \mathrm{O},{ }^{20} \mathrm{O}\right.$, and $\left.{ }^{22} \mathrm{O}\right)$ impinging on a carbon target at energies between $350 \mathrm{~A}$ and $450 \mathrm{~A} \mathrm{MeV}$. The inverse kinematic condition, together with the large acceptance of the experimental setup, allowed us to determine these cross sections with minimum corrections and, therefore, good accuracy.

Many of the cross sections obtained with stable projectiles have been previously measured in other works at similar energies. The measurements performed by Webber and collaborators [10] for the three stable projectiles, at a slightly higher energy, $600 \mathrm{~A} \mathrm{MeV}$, are in rather good agreement with our results. For 25 out of 30 common measurements the final cross sections deviate less than $15 \%$, and for the other four less than $30 \%$. Only for ${ }^{7} \mathrm{Be}$, produced in reactions induced by ${ }^{14} \mathrm{~N}$ on carbon, we observe a large discrepancy.

A very good agreement was also observed for the production cross section of ${ }^{11} \mathrm{~B}$ in reactions induced by ${ }^{12} \mathrm{C}$ projectiles on a carbon target at almost the same energy obtained by Kidd et al. [9] and more recently by Panin et al. [43].

Ogawa and collaborators performed a few years ago a detailed investigation of the fragmentation residues produced in reactions ${ }^{12} \mathrm{C}$ on $\mathrm{C}$ at energies between $100 \mathrm{~A}$ and $400 \mathrm{~A}$ $\mathrm{MeV}$ [11]. The measurements obtained in this paper for the highest investigated energies show significantly smaller cross sections. This is particularly true for the boron and beryllium isotopes.

We also used this extended set of data to benchmark some of the most widely used nuclear reactions codes. In particular, we confronted the measured cross sections with predictions obtained with the abrasion-ablation code ABRABLA07 [28,63], the Liège intranuclear cascade code INCL [31] coupled to the ablation code ABLA07 [63], and the INCL code couple to the Fermi breakup model [66].
Although the three calculations provide reasonable overall description of the data, the most accurate prediction is the one obtained with the abrasion-ablation code ABRABLA07. Indeed, this code reproduces most of the measured cross sections with an accuracy better than $20 \%$. We only observed relevant deviation for the single-neutron removal channels in reactions induced by neutron-rich oxygen isotopes $\left({ }^{20} \mathrm{O}\right.$ and ${ }^{22} \mathrm{O}$ ). Other observed deviations correspond to residual fragments produced in the removal of more than eight nucleons, and could be due to an overestimation of the geometrical acceptance used to evaluate the measured cross sections. Interestingly, the present predictions were obtained with the standard version of the abrasion model without any modification in the excitation energy as required for medium-mass or heavy projectile nuclei [21], or deduced in the analysis of few nucleon removal channels for the same reactions [44].

The INCL model coupled to the deexcitation ABLA07 code also provides a satisfactory description of many of the measured cross sections, even though this code has been originally developed to describe reactions induced by relativistic protons on medium-mass and heavy nuclei. Indeed, the extended version of the code to describe ion-ion collisions failed in describing the cross sections of fragments produced in reactions induced by neutron-rich oxygen isotopes $\left({ }^{20} \mathrm{O}\right.$ and ${ }^{22} \mathrm{O}$ ) while the standard version for reactions induced by protons provided reasonable results. The main disagreements concern the most peripheral reactions. We observe a general overprediction of single-proton removal channels in reactions induced by neutron-deficient projectiles, and an underprediction of single-neutron removal channels in reactions induced by neutron-rich projectiles. Given that INCL includes a realistic description of the neutron and proton density profiles [33], the observed discrepancy could be attributed to other parameters that also play important roles in the description of ion-ion collisions such as the Pauli blocking or an inaccurate determination of the dynamical quantities (e.g., atomic and mass numbers, excitation energy, etc.) during the projectile and target remnant thermalization process [31].

On the other hand, predictions obtained with the INCL code coupled to the Fermi breakup model provided also a reasonable description of the cross sections within the range of fragments covered by the Fermi breakup $(A<18)$. We only observe a certain overestimation of the one-neutron removal channel together with an underestimation of the one-proton removal channels. A surprising result was a clear underestimation of the production cross section of ${ }^{12} \mathrm{C}$ in some of the investigated reactions. This result was tentatively attributed to the fact that the Fermi breakup model does not use evaluated masses but calculated ones according to the liquiddrop model.

Finally, advanced reaction models describing the dynamics and correlations of the fragmentation process such as quantum molecular dynamics [80], including advanced cluster recognition algorithms [81], Boltzmann-Langevin one body [82], or antisymmetrized molecular dynamics [83] approaches, may provide a deeper insight into the production of final residues in the range covered by the present paper. 


\section{ACKNOWLEDGMENTS}

This work has been partially supported by the Spanish Ministry for Science and Innovation under Grants No. PGC2018-099746-B-C21, No. PGC2018-099746-B-C22, and No. PID2019-104390GB-100; Xunta de Galicia under program "Grupos de referencia competitiva" (Project No. ED431C 2017/54); the German Bundesministerium für Bildung und Forschung (BMBF) (Grants No. 05P12RDFN8, No. 05P15RDFN1, and No. 05P19RDFN); the Swedish Research Council; and U.S. Department of Energy Grant No. DE-FG02-08ER41533. J.J.R.S. acknowledges the support of Xunta de Galicia under Grant No. ED481B-2017/002.

\section{APPENDIX: MEASURED PRODUCTION CROSS SECTIONS}

Table I presents the 135 cross sections obtained in this paper.

TABLE I. Isotopic production cross sections of residual nuclei produced in the fragmentation reactions induced by light stable and unstable projectiles impinging a carbon target investigated in this paper. Each reaction channel is characterized by the $(Z, A)$ numbers for projectiles (subscript $p$ ) and final fragments $(f)$. Final errors including systematic and statistical uncertainties are indicated.

\begin{tabular}{|c|c|c|c|}
\hline$E(\mathrm{MeV})$ & $(Z, A)_{p}$ & $(Z, A)_{f}$ & $\sigma_{\text {frag }}(\mathrm{mb})$ \\
\hline $397 A$ & 8,13 & $\begin{array}{c}7,12 \\
6,12 \\
6,11 \\
6,10 \\
6,9 \\
5,11 \\
5,10 \\
5,8 \\
4,9 \\
4,7\end{array}$ & $\begin{aligned} 21.56 & \pm 3.34 \\
4.56 & \pm 1.17 \\
76.08 & \pm 5.14 \\
40.79 & \pm 3.92 \\
13.13 & \pm 2.06 \\
1.28 & \pm 0.49 \\
12.48 & \pm 1.90 \\
9.04 & \pm 1.55 \\
1.08 & \pm 0.44 \\
16.35 & \pm 2.39\end{aligned}$ \\
\hline $349 A$ & 8,14 & $\begin{array}{c}7,13 \\
7,12 \\
7,11 \\
6,12 \\
6,11 \\
6,10 \\
6,9 \\
5,11 \\
5,10 \\
5,10 \\
4,10 \\
4,9 \\
4,7\end{array}$ & $\begin{array}{r}38.65 \pm 3.01 \\
14.29 \pm 1.50 \\
0.24 \pm 0.24 \\
60.41 \pm 3.36 \\
66.35 \pm 4.65 \\
16.88 \pm 2.17 \\
2.85 \pm 1.00 \\
7.45 \pm 1.05 \\
12.20 \pm 1.40 \\
27.72 \pm 3.20 \\
0.25 \pm 0.16 \\
2.93 \pm 0.70 \\
7.29 \pm 2.25\end{array}$ \\
\hline $308 A$ & 8,15 & $\begin{array}{l}8,14 \\
8,13 \\
7,14 \\
7,13 \\
7,12 \\
6,13 \\
6,12 \\
6,11\end{array}$ & $\begin{aligned} 11.08 & \pm 2.01 \\
3.33 & \pm 1.57 \\
65.38 & \pm 6.06 \\
32.25 & \pm 4.15 \\
5.84 & \pm 2.04 \\
24.50 & \pm 3.34 \\
60.28 & \pm 5.78 \\
41.51 & \pm 4.63\end{aligned}$ \\
\hline
\end{tabular}

TABLE I. (Continued).

\begin{tabular}{|c|c|c|c|}
\hline$E(\mathrm{MeV})$ & $(Z, A)_{p}$ & $(Z, A)_{f}$ & $\sigma_{\text {frag }}(\mathrm{mb})$ \\
\hline & & 6,10 & $3.91 \pm 1.41$ \\
\hline & & 6,9 & $1.00 \pm 1.02$ \\
\hline & & 5,11 & $9.97 \pm 1.83$ \\
\hline & & 5,10 & $12.12 \pm 2.09$ \\
\hline & & 4,10 & $1.62 \pm 0.82$ \\
\hline & & 4,9 & $10.21 \pm 3.05$ \\
\hline & & 4,7 & $3.77 \pm 3.85$ \\
\hline \multirow[t]{15}{*}{$450 A$} & 8,16 & 8,15 & $69.60 \pm 3.91$ \\
\hline & & 8,14 & $3.21 \pm 0.46$ \\
\hline & & 7,15 & $60.11 \pm 3.38$ \\
\hline & & 7,14 & $49.37 \pm 2.84$ \\
\hline & & 7,12 & $0.73 \pm 0.26$ \\
\hline & & 6,14 & $5.59 \pm 0.54$ \\
\hline & & 6,13 & $19.72 \pm 1.85$ \\
\hline & & 6,12 & $49.73 \pm 4.01$ \\
\hline & & 6,11 & $16.850 \pm 1.92$ \\
\hline & & 6,10 & $1.43 \pm 0.37$ \\
\hline & & 5,13 & $0.19 \pm 0.07$ \\
\hline & & 5,11 & $18.22 \pm 1.60$ \\
\hline & & 5,10 & $15.82 \pm 1.83$ \\
\hline & & 4,10 & $0.99 \pm 0.20$ \\
\hline & & 4,9 & $3.89 \pm 0.60$ \\
\hline \multirow[t]{25}{*}{$415 A$} & 8,20 & 8,19 & $92.94 \pm 4.71$ \\
\hline & & 8,18 & $69.49 \pm 3.51$ \\
\hline & & 8,17 & $27.02 \pm 1.37$ \\
\hline & & 8,16 & $15.31 \pm 0.78$ \\
\hline & & 8,15 & $4.48 \pm 0.39$ \\
\hline & & 7,19 & $21.68 \pm 1.12$ \\
\hline & & 7,18 & $18.63 \pm 0.95$ \\
\hline & & 7,17 & $37.46 \pm 1.89$ \\
\hline & & 7,16 & $19.85 \pm 1.01$ \\
\hline & & 7,15 & $40.40 \pm 2.04$ \\
\hline & & 7,14 & $12.14 \pm 0.93$ \\
\hline & & 7,13 & $1.40 \pm 0.18$ \\
\hline & & 6,16 & $4.52 \pm 0.25$ \\
\hline & & 6,15 & $12.06 \pm 0.92$ \\
\hline & & 6,14 & $29.91 \pm 2.26$ \\
\hline & & 6,13 & $22.15 \pm 2.22$ \\
\hline & & 6,12 & $19.74 \pm 2.97$ \\
\hline & & 5,14 & $1.54 \pm 0.17$ \\
\hline & & 5,13 & $5.82 \pm 0.32$ \\
\hline & & 5,12 & $9.45 \pm 0.73$ \\
\hline & & 5,11 & $21.03 \pm 2.12$ \\
\hline & & 5,10 & $2.15 \pm 0.40$ \\
\hline & & 4,12 & $1.41 \pm 0.23$ \\
\hline & & 4,11 & $3.13 \pm 0.52$ \\
\hline & & 4,10 & $6.93 \pm 1.41$ \\
\hline \multirow[t]{9}{*}{$414 A$} & 8,22 & 8,21 & $87.67 \pm 4.53$ \\
\hline & & 8,20 & $70.43 \pm 3.55$ \\
\hline & & 8,19 & $38.96 \pm 2.07$ \\
\hline & & 8,18 & $38.90 \pm 1.81$ \\
\hline & & 8,17 & $14.31 \pm 1.05$ \\
\hline & & 8,16 & $4.51 \pm 0.46$ \\
\hline & & 7,21 & $16.12 \pm 1.10$ \\
\hline & & 7,20 & $23.91 \pm 1.30$ \\
\hline & & 7,19 & $37.59 \pm 2.30$ \\
\hline
\end{tabular}


TABLE I. (Continued).

\begin{tabular}{ccc}
\hline \hline$E(\mathrm{MeV})$ & $(Z, A)_{f}$ & $\sigma_{\text {frag }}(\mathrm{mb})$ \\
\hline & 7,18 & $29.54 \pm 1.63$ \\
7,17 & $38.17 \pm 2.70$ \\
7,16 & $24.87 \pm 2.57$ \\
7,15 & $40.32 \pm 6.37$ \\
& 7,14 & $4.95 \pm 0.94$ \\
& 6,19 & $0.30 \pm 0.10$ \\
& 6,18 & $4.63 \pm 0.68$ \\
& 6,17 & $4.80 \pm 0.56$ \\
& 6,16 & $11.89 \pm 1.58$ \\
& 6,15 & $12.56 \pm 1.30$ \\
& 6,14 & $35.28 \pm 4.63$ \\
& 6,13 & $24.56 \pm 5.35$ \\
& 6,12 & $4.07 \pm 0.71$ \\
& 5,15 & $1.54 \pm 0.22$ \\
& 5,14 & $1.76 \pm 0.29$ \\
& 5,13 & $11.84 \pm 1.65$ \\
& 5,12 & $7.36 \pm 1.44$ \\
& 5,11 & $8.19 \pm 2.02$ \\
& 4,11 & $0.27 \pm 0.15$ \\
& 4,10 & $2.35 \pm 0.66$ \\
& 4,9 & $1.66 \pm 0.66$ \\
& 6,11 & $65.96 \pm 5.48$ \\
& 6,10 & $4.01 \pm 0.64$ \\
& &
\end{tabular}

TABLE I. (Continued).

\begin{tabular}{|c|c|c|c|}
\hline$E(\mathrm{MeV})$ & $(Z, A)_{p}$ & $(Z, A)_{f}$ & $\sigma_{\text {frag }}(\mathrm{mb})$ \\
\hline & & 5,11 & $59.48 \pm 2.17$ \\
\hline & & 5,10 & $36.65 \pm 2.30$ \\
\hline & & 5,8 & $0.84 \pm 0.16$ \\
\hline & & 4,10 & $6.21 \pm 0.61$ \\
\hline & & 4,9 & $10.37 \pm 1.17$ \\
\hline & & 4,7 & $12.83 \pm 2.26$ \\
\hline & & 3,8 & $1.66 \pm 0.32$ \\
\hline & & 3,7 & $15.63 \pm 4.19$ \\
\hline \multirow[t]{15}{*}{$450 A$} & 7,14 & 7,13 & $16.16 \pm 1.31$ \\
\hline & & 7,12 & $1.61 \pm 0.34$ \\
\hline & & 6,14 & $0.79 \pm 0.23$ \\
\hline & & 6,13 & $18.12 \pm 1.40$ \\
\hline & & 6,12 & $104.11 \pm 5.91$ \\
\hline & & 6,11 & $23.66 \pm 2.19$ \\
\hline & & 6,10 & $1.02 \pm 0.28$ \\
\hline & & 5,12 & $3.20 \pm 0.46$ \\
\hline & & 5,11 & $32.18 \pm 2.20$ \\
\hline & & 5,10 & $27.72 \pm 3.20$ \\
\hline & & 5,8 & $0.78 \pm 0.44$ \\
\hline & & 4,11 & $0.15 \pm 0.09$ \\
\hline & & 4,10 & $1.82 \pm 0.35$ \\
\hline & & 4,9 & $5.37 \pm 0.82$ \\
\hline & & 4,7 & $2.19 \pm 0.64$ \\
\hline
\end{tabular}

[1] M. V. Ricciardi, A. V. Ignatyuk, A. Kelić, P. Napolitani, F. Rejmund, K.-H. Schmidt, and O. Yordanov, Nucl Phys. A 733, 299 (2004).

[2] P. Russotto et al., Phys. Rev. C 94, 034608 (2016).

[3] J. Pochodzalla, T. Mohlenkamp, T. Rubehn, A. Schottauf, A. Worner, E. Zude, M. Begemann-Blaich, T. Blaich, H. Emling, A. Ferrero, C. Gross, G. Imme, I. Iori, G. J. Kunde, W. D. Kunze, V. Lindenstruth, U. Lynen, A. Moroni, W. F. J. Muller, B. Ocker, G. Raciti, H. Sann, C. Schwarz, W. Seidel, V. Serving, J. Stroth, W. Trautmann, A. Trzcinski, A. Tucholski, G. Verde, and B. Zwieglinski, Phys. Rev. Lett. 75, 1040 (1995).

[4] H. Alvarez-Pol, J. Benlliure, E. Casarejos, L. Audouin, D. Cortina-Gil, T. Enqvist, B. Fernandez-Dominguez, A. R. Junghans, B. Jurado, P. Napolitani, J. Pereira, F. Rejmund, K. H. Schmidt, and O. Yordanov, Phys. Rev. C 82, 041602(R) (2010).

[5] T. Saito et al., Nucl. Phys. A 881, 218 (2012).

[6] C. Zeitlin and C. La Tessa, Front. Oncol. 6, 65 (2016).

[7] W. D. Newhauser and M. Durante, Nat. Rev. Cancer 11, 438 (2011).

[8] J. W. Norbury et al., Life. Sci. Space Res. (Amst). 8, 38 (2016).

[9] J. M. Kidd, P. J. Lindstrom, H. J. Crawford, and G. Woods, Phys. Rev. C 37, 2613 (1988).

[10] W. R. Webber, J. C. Kish, and D. A. Schrier, Phys. Rev. C 41, 547 (1990).

[11] T. Ogawa, T. Sato, S. Hashimoto, D. Satoh, S. Tsuda, and K. Niita, Phys. Rev. C 92, 024614 (2015).

[12] I. Schall et al., Nucl. Instrum. and Methods B 117, 221 (1996).

[13] A. N. Golovchenko, J. Skvarc, N. Yasuda, M. Giacomelli, S. P. Tretyakova, R. Ilic, R. Bimbot, M. Toulemonde, and T. Murakami, Phys. Rev. C 66, 014609 (2002).

[14] T. Toshito, K. Kodama, L. Sihver, K. Yusa, M. Ozaki, K. Amako, S. Kameoka, K. Murakami, T. Sasaki, S. Aoki, T.
Ban, T. Fukuda, M. Komatsu, H. Kubota, N. Naganawa, T. Nakamura, T. Nakano, M. Natsume, K. Niwa, S. Takahashi, J. Yoshida, H. Yoshida, M. Kanazawa, N. Kanematsu, M. Komori, S. Sato, M. Asai, T. Koi, C. Fukushima, S. Ogawa, M. Shibasaki, and H. Shibuya, Phys. Rev. C 75, 054606 (2007).

[15] C. Zeitlin, S. Guetersloh, L. Heilbronn, J. Miller, A. Fukumura, Y. Iwata, and T. Murakami, Phys. Rev. C 76, 014911 (2007).

[16] C. Zeitlin, J. Miller, S. Guetersloh, L. Heilbronn, A. Fukumura, Y. Iwata, T. Murakami, S. Blattnig, R. Norman, and S. Mashnik, Phys. Rev. C 83, 034909 (2011).

[17] Y. P. Viyogi, T. J. M. Symons, P. Doll, D. E. Greiner, H. H. Heckman, D. L. Hendrie, P. J. Lindstrom, J. Mahoney, D. K. Scott, K. VanBibber, G. D. Westfall, H. Wieman, H. J. Crawford, C. McParland, and C. K. Gelbke, Phys. Rev. Lett. 42, 33 (1979).

[18] M. Mocko, M. B. Tsang, L. Andronenko, M. Andronenko, F. Delaunay, M. Famiano, T. Ginter, V. Henzl, D. Henzlova, H. Hua, S. Lukyanov, W. G. Lynch, A. M. Rogers, M. Steiner, A. Stolz, O. Tarasov, M. J. vanGoethem, G. Verde, W. S. Wallace, and A. Zalessov, Phys. Rev. C 74, 054612 (2006).

[19] O. B. Tarasov, M. Portillo, D. J. Morrissey, A. M. Amthor, L. Bandura, T. Baumann, D. Bazin, J. S. Berryman, B. A. Brown, G. Chubarian, N. Fukuda, A. Gade, T. N. Ginter, M. Hausmann, N. Inabe, T. Kubo, J. Pereira, B. M. Sherrill, A. Stolz, C. Sumithrarachichi, M. Thoennessen, and D. Weisshaar, Phys. Rev. C 87, 054612 (2013).

[20] J. Benlliure, M. Fernandez-Ordonez, L. Audouin, A. Boudard, E. Casarejos, J. E. Ducret, T. Enqvist, A. Heinz, D. Henzlova, V. Henzl, A. Kelic, S. Leray, P. Napolitani, J. Pereira, F. Rejmund, M. V. Ricciardi, K. H. Schmidt, C. Schmitt, C. Stephan, L. Tassan-Got, C. Volant, C. Villagrasa, and O. Yordanov, Phys. Rev. C 78, 054605 (2008). 
[21] J. Benlliure, K.-H. Schmidt, D. Cortina-Gil, T. Enqvist, F. Farget, A. Heinz, A. R. Junghans, J. Pereira, and J. Taieb, Nucl. Phys. A 660, 87 (1999).

[22] M. de Jong et al., Nucl. Phys. A 628, 479 (1998).

[23] T. Enqvist, J. Benlliure, F. Farget, K.-H. Schmidt, P. Armbruster, M. Bernas, L. Tassan-Got, A. Boudard, R. Legrain, C. Volant, C. Böckstiegel, M. de Jong, and J. P. Dufour, Nucl. Phys. A 658, 47 (1999).

[24] D. Pérez-Loureiro et al., Phys. Lett. B 703, 552 (2011).

[25] H. Wang et al., Phys. Lett. B 754, 104 (2016).

[26] P. G. Hansen and J. A. Tostevin, Ann. Rev. Nucl. Part. Scien. 53, 219 (2003).

[27] R. Serber, Phys. Rev. 72, 1114 (1947).

[28] J. J. Gaimard and K.-H. Schmidt, Nucl. Phys. A 531, 709 (1991).

[29] A. Boudard, J. Cugnon, S. Leray, and C. Volant, Phys. Rev. C 66, 044615 (2002).

[30] K.-H. Schmidt et al., Phys. Lett. B 300, 313 (1993).

[31] D. Mancusi, A. Boudard, J. Cugnon, J.-C. David, P. Kaitaniemi, and S. Leray, Phys. Rev. C 90, 054602 (2014).

[32] T. Kurtukian-Nieto et al., Phys. Rev. C 89, 024616 (2014).

[33] J. L. Rodríguez-Sánchez, J.-C. David, D. Mancusi, A. Boudard, J. Cugnon, and S. Leray, Phys. Rev. C 96, 054602 (2017).

[34] J. L. Rodríguez-Sánchez et al., Phys. Rev. C 96, 034303 (2017).

[35] J. Díaz-Cortés et al., Phys. Lett. B 811, 135962 (2020).

[36] B. Borderie and M. F. Rivet, Prog. Part. Nucl. Phys. 61, 551 (2008).

[37] A. Schüttauf et al., Nucl. Phys. A 607, 457 (1996).

[38] W. Trautmann et al., Prog. Part. Nucl. Phys. 62, 407 (2009).

[39] T. Aumann et al., Prog. Part. Nucl. Phys. 59, 3 (2007).

[40] C. Caesar et al. ( $\mathrm{R}^{3} \mathrm{~B}$ Collaboration), Phys. Rev. C 88, 034313 (2013).

[41] F. Wamers et al. ( $\mathrm{R}^{3} \mathrm{~B}$ Collaboration), Phys. Rev. Lett. 112, 132502 (2014).

[42] J. Marganiec et al. ( $\mathrm{R}^{3} \mathrm{~B}$ Collaboration), Eur. Phys. J. A 51, 9 (2015).

[43] V. Panin et al., Phys. Lett B 753, 204 (2016).

[44] R. Thies et al. ( $\mathrm{R}^{3} \mathrm{~B}$ Collaboration), Phys. Rev. C 93, 054601 (2016).

[45] M. Röder et al. ( $\mathrm{R}^{3} \mathrm{~B}$ Collaboration), Phys. Rev. C 93, 065807 (2016).

[46] M. Heine et al. ( $\mathrm{R}^{3} \mathrm{~B}$ Collaboration), Phys. Rev. C 95, 014613 (2017).

[47] M. Vandebrouck et al. ( $\mathrm{R}^{3} \mathrm{~B}$ Collaboration), Phys. Rev. C 96, 054305 (2017).

[48] P. Díaz Fernández et al. ( $\mathrm{R}^{3} \mathrm{~B}$ Collaboration), Phys. Rev. C 97, 024311 (2018).

[49] L. Atar et al. ( $\mathrm{R}^{3} \mathrm{~B}$ Collaboration), Phys. Rev. Lett. 120, 052501 (2018).

[50] F. Wamers et al. ( $\mathrm{R}^{3} \mathrm{~B}$ Collaboration), Phys. Rev. C 97, 034612 (2018).

[51] M. Holl et al. ( $\mathrm{R}^{3} \mathrm{~B}$ Collaboration), Phys. Lett. B 795, 682 (2019).

[52] I. Syndikus et al., Phys. Lett. B 809, 135748 (2020).

[53] H. Geissel et al., Nucl. Instr. Methods Phys. Res., Sect. B 70, 286 (1992).

[54] V. Metag et al., Nucl. Phys. A 409, 331 (1983).

[55] K. Mahata et al., Nucl. Instrum. Methods Phys. Res., Sect. A 608, 331 (2009).

[56] V. T. Blaich et al., Nucl. Instrum. Methods Phys. Res., Sect. A 314, 136 (1992).
[57] P. J. Karol, Phys. Rev. C 11, 1203 (1975).

[58] V. Panin et al., Phys. Lett. B 797, 134802 (2019).

[59] F. Grenier et al., Nucl. Phys. A 811, 233 (2008).

[60] A. Kelić, K. H. Schmidt, T. Enqvist, A. Boudard, P. Armbruster, J. Benlliure, M. Bernas, S. Czajkowski, R. Legrain, S. Leray, B. Mustapha, M. Pravikoff, F. Rejmund, C. Stéphan, J. Taïeb, L. Tassan-Got, C. Volant, and W. Wlazlo, Phys. Rev. C 70, 064608 (2004).

[61] J. L. Rodríguez-Sánchez et al., Phys. Lett. B 807, 135565 (2020)

[62] R. J. Glauber and G. Matthiae, Nucl. Phys. B 21, 135 (1970).

[63] A. Kelić, M. V. Ricciardi, and K.-H. Schmidt, in Proceedings of Joint ICTP-IAEA Advanced Workshop on Model Codes for Spallation Reactions, ICTP Trieste, Italy, 4-8 February 2008, edited by D. Filges, S. Leray, Y. Yariv, A. Mengoni, A. Stanculescu, and G. Mank, IAEA Conf. Proc. No. INDC(NDS)-530 (IAEA, Vienna, 2008), pp. 181-221.

[64] V. F. Weisskopf and D. H. Ewing, Phys. Rev. 57, 472 (1940).

[65] K.-H. Schmidt, M. V. Ricciardi, A. S. Botvina, and T. Enqvist, Nucl. Phys. A 710, 157 (2002).

[66] E. Fermi, Prog. Theor. Phys. 5, 570 (1950).

[67] L. F. Oliveira, R. Donangelo, and J. O. Rasmussen, Phys. Rev. C 19, 826 (1979).

[68] A. Boudard, J. Cugnon, J.-C. David, S. Leray, and D. Mancusi, Phys. Rev. C 87, 014606 (2013).

[69] J. Cugnon, D. L'Hôte, and J. Vandermeulen, Nucl. Instr. Methods Phys. Res., Sect. B 111, 215 (1996).

[70] J. Cugnon and P. Henrotte, Eur. Phys. J. A 16, 393 (2003).

[71] B. Jurado, K.-H. Schmidt, and J. Benlliure, Phys. Lett. B 553, 186 (2003).

[72] J. Benlliure et al., Nucl. Phys. A 628, 458 (1998).

[73] M. Wang, G. Audi, F. G. Kondev, W. J. Huang, S. Naimi, and X. Xu, Chin. Phys. C 41, 030003 (2016).

[74] R. Bass, Proceedings of the Symposium on Deep-Inelastic and Fusion Reactions with Heavy Ions (Springer-Verlag, Berlin, 1980).

[75] J. B. Natowitz, R. Wada, K. Hagel, T. Keutgen, M. Murray, A. Makeev, L. Qin, P. Smith, and C. Hamilton, Phys. Rev. C 65, 034618 (2002).

[76] C. Sfienti et al., Nucl. Phys. A 787, 627 (2007).

[77] A. S. Botvina, N. Buyukcizmeci, M. Erdogan, J. Łukasik, I. N. Mishustin, R. Ogul, and W. Trautmann, Phys. Rev. C 74, 044609 (2006).

[78] A. S. Botvina et al., Nucl. Phys. A 475, 663 (1987).

[79] L. Audirac, A. Obertelli, P. Doornenbal, D. Mancusi, S. Takeuchi, N. Aoi, H. Baba, S. Boissinot, A. Boudard, A. Corsi, A. Gillibert, T. Isobe, A. Jungclaus, V. Lapoux, J. Lee, S. Leray, K. Matsui, M. Matsushita, T. Motobayashi, D. Nishimura, S. Ota, E. C. Pollacco, G. Potel, H. Sakurai, C. Santamaria, Y. Shiga, D. Sohler, D. Steppenbeck, R. Taniuchi, and H. Wang, Phys. Rev. C 88, 041602(R) (2013).

[80] J. Aichelin, Phys. Rep. 202, 233 (1991).

[81] A. Le Fèvre, J. Aichelin, C. Hartnack, and Y. Leifels, Phys. Rev. C 100, 034904 (2019).

[82] P. Napolitani and M. Colonna, Phys. Lett. B 726, 383 (2013).

[83] Y. Kanada-En'yo, M. Kimura, and A. Ono, Prog. Theor. Exp. Phys. 1, 01A202 (2012).

Correction: Affiliation address 19 was incomplete and has been fixed. 